\title{
Electrically induced monosynaptic reflexes in man
}

\author{
E. DIAMANTOPOULOS AND M. M. GASSEL \\ From the department of Clinical Neurophysiology, University of Freiburg, Germany
}

A monosynaptic reflex produced by electrical stimulation of the mixed nerve in humans (H-reflex) can frequently be evoked in the calf muscles at a stimulus intensity below threshold for the motor nerve fibres. In special states, such as spasticity, low threshold $\mathrm{H}$-reflexes are more easily elicited and more widely distributed in muscles other than the calf (Teasdall, Park, Languth, and Magladery, 1952; Bolzani and Sala, 1958; Pinelli and Valle, 1960). It has been reported that centripetal nerve volley can sometimes be recorded below threshold for the direct motor response on stimulation of various peripheral nerves in the upper and lower extremity (Dawson and Scott, 1949; Gilliatt, Goodman, and Willison, 1961) in whose distribution an H-reflex is an unusual occurrence. Yet Magladery, Teasdall, Park, and Porter (1951b), using intrathecal electrodes and recording from the spinal roots on stimulation of the posterior tibial nerve, found that the afferent volley did not appear until the reflex volley was well developed. One purpose of the present study was to investigate the relationship between centripetal nerve volleys and the threshold for the development of the monosynaptic reflex in the calf and other muscles, in normal subjects and in spastic patients.

The afferent limb of the two-neurone arc is transmitted in the largest proprioceptive fibres (group 1a) and the efferent limb in alpha motor neurones. The absolute and relative conduction velocity of the sensory limb of the monosynaptic reflex and that of the alpha motor fibres has been studied by methods which are diverse and with results which are at variance (Magladery and McDougal, 1950; Liberson, 1962; Mayer, 1963; Angel and Alston, 1964; Magladery, Porter, Park, and Teasdall, 1951a). A further purpose of this study was to investigate the conduction velocity of the afferent limb of the monosynaptic reflex in the calf muscles over the long stretch of tibial nerve from the popliteal region to the upper thigh, directly by recording the afferent volley, and indirectly by changes in latency of the H-reflex elicited at proximal and distal sites; the conduction velocity of the motor fibres was also determined, by changes in latency of the direct motor response evoked at the same sites.

'Supported by a grant from the Deutsche Forschungsgemeinschaft.
METHODS AND MATERIALS

Twenty-four normal subjects, and six patients with spasticity, the result of various cerebral diseases, were investigated. The ages of the normal subjects ranged from 18 to 67 years, and those of the patients from 30 to 60 years.

The subjects were placed in the prone position with the feet extending over the end of the couch. Recordings of the muscle action potentials from the calf muscles were made with non-insulated subcutaneous needle electrodes fixed in place over the muscle belly and tendon. The nerve action potentials were recorded from the tibial nerve in the upper popliteal fossa and from the sciatic nerve in the upper thigh using bipolar, non-insulated, steel needle electrodes placed $2.0 \mathrm{~cm}$. apart. In the upper popliteal region the area for the lowest threshold for ad $\overrightarrow{0}$ response in the calf muscles was first determined wit $\bar{D}$. bipolar surface stimulating electrodes with a diameter of $6 \mathrm{~mm}$. for each electrode and a centre-to-centre separat tion of $25 \mathrm{~mm}$. The needle electrodes, $34 \cdot 3 \mu$ in externaf diameter and $4 \mathrm{~cm}$. in length, were then inserted in the same position along the course of the nerve. In the upper? thigh the position of the sciatic nerve was determine by surface anatomy; and the needles, $90.6 \mu$ in diameter and $8 \mathrm{~cm}$. in length, were inserted directly below the middle of the greater trochanter of the femur and the tuberosity of the ischium on a line drawn downwards. to the apex of the popliteal fossa (Gassel and Trojaborg, 1964). The proximity of the needle electrodes to the nerve was determined by using the needles as stimulating electrodes and finding the position of lowest threshold for a response in the triceps surae. Slight adjustments in position of the electrodes were frequently necessary, and it was usually possible to evoke a very low threshold response, and a response which greatly increased with a slight increment in intensity of the stimulus. Paraesthesiae extending into the feet were frequently experienced on insertion of the electrodes and were generally a good index of satisfactory positioning of the needles. Care was taken at both sites to avoid stimulation of the lateral popliteal nerve; at the sciatic site the lateral popliteal nerve was frequently co-stimulated, but at high intensity stimulation.

The posterior tibial nerve was stimulated with the bipolar surface electrodes in the low popliteal region. A thin flat lead plate ground, $3 \times 8 \mathrm{~cm}$., was placed between the stimulating and recording electrodes. The stimulus was a rectangular pulse $0.5 \mathrm{msec}$. in duration delivered from a stimulator with transformer output. The output impedance was $10 \mathrm{kilohms}$. The frequency of stimulation was one every two seconds. 
The stimulus intensity was increased in stages from that subthreshold for the reflex and direct motor response to that producing an H-reflex, but subthreshold for the direct motor response, and with the development of the direct motor response.

A trigger pulse initiated both the sweep and the stimulus. The Tönnies electromyograph was used for recording. This apparatus has three D.C. coupled amplifiers, each with an input impedance of 4 megohms. Time constant was $30 \mathrm{msec}$. The nerve action potentials were amplified and displayed on a single beam oscilloscope. Single interrupted sweeps were taken with a time base of either $0.5,1.0$, or $1.5 \mathrm{msec}$. per $\mathrm{mm}$. The muscle action potentials from the triceps surae were differentially amplified and simultaneously displayed on one beam of a double-beam oscilloscope; the time base was $20 \mathrm{msec}$. $/ \mathrm{mm}$. Twenty photographic recordings were made of the responses at each increment of stimulus.

The latency was measured to the start of the nerve action potentials. The conduction velocity was calculated by dividing the distance between the centre of each pair of recording needles by the difference in latency of the responses at each site. Conduction velocity was also determined by the latency of the response at the sciatic recording electrodes from the stimulating cathode in the popliteal region. This latency was measured from the start of the sweep and $0.5 \mathrm{msec}$. was added to adjust for the duration of the stimulus pulse whose onset was not recorded.

In all measurements of conduction velocity complexes were utilized in which the amplification of the potential was adequate to give clear definition of the start. The results are based upon these selected samples. Other features of the procedure will be expanded in the Results.

Recording for nerve potentials was also made from the upper trunk of the brachial plexus in the supraclavicular region (Erb's point) on stimulation of the musculocutaneous nerve in the upper arm. The position of the upper trunk of the brachial plexus was first localized by determining the lowest threshold of response in the biceps brachii muscle on stimulation with the surface electrode (Gassel, 1964). Subcutaneous needle electrodes were then fixed in place in the corresponding positions. The needles were non-insulated, stainless steel, $40.5 \mu$ in external diameter and $3 \mathrm{~cm}$. in length, and with the distal $5 \mathrm{~mm}$. bent at right angles for insertion. Subcutaneous recording electrodes were placed over the belly and tendon of the biceps brachii. A response was elicited limited to the biceps brachii on stimulation of the musculocutaneous nerve in the upper arm using surface stimulating electrodes. The nerve action potentials were recorded at stages during increments in stimulus intensity, in a manner analogous to that described above.

The H-reflex and direct motor response in the triceps surae were elicited on stimulation of the posterior tibial nerve in the popliteal fossa and the sciatic nerve in the upper thigh. The needle electrodes used for recording of nerve potentials were generally utilized for eliciting the H-reflex and direct motor response; however, some change in the position of the needles was generally necessary to obtain a large H-reflex with little or no direct motor response, and complexes which were of the same ampli- tude and similar appearance at the two sites. The distance between the stimulating cathodes in the popliteal and sciatic regions was divided by the difference in latency of the direct motor responses, and of the H-reflexes, in determining the conduction velocity of the alpha motor fibres and of the afferent limb of the H-reflex, respectively. It was not always possible to evoke low threshold reflexes of similar appearance at the sciatic and popliteal sites, and the results are based upon those instances in which these criteria were satisfied.

The temperature of the room in which the test was performed was $21-23^{\circ} \mathrm{C}$.

The procedure was well tolerated, and there were no untoward complications.

RESULTS

THRESHOLDS FOR THE H-REFLEX AND DIRECT MOTOR RESPONSE It was frequently possible to evoke the reflex response in the calf muscles on stimulation of the posterior tibial nerve in the popliteal region at a threshold below that for the direct motor response. However, considerable changes in position of the stimulating electrodes were sometimes necessary to achieve this result and it was an almost constant observation that by slightly altering the position of the stimulating electrodes the direct motor response could be elicited at a threshold below that of the reflex response. It was sometimes impossible to elicit a reflex response subthreshold for the direct motor response, and this was most frequent in older subjects with weak tendon reflexes, whereas $\mathbf{H}$ reflexes of large amplitude and low threshold were more easily elicited in subjects with brisk tendon reflexes and especially in spastic patients. In this latter group of subjects reflexes subthreshold for the direct motor response were sometimes also found in other muscles, as in the quadriceps femoris on stimulation of the femoral nerve, or in the biceps brachii on stimulation of the upper trunk of the brachial plexus.

NERVE POTENTIALS RECORDED ON ELECTRICAL STIMULATION OF A MIXED PERIPHERAL NERVE IN RELATION TO THE DEVELOPMENT OF THE H-REFLEX AND DIRECT MOTOR RESPONSE Nerve potentials were recorded from the high popliteal region and upper thigh on stimulation of the posterior tibial nerve in 11 subjects, and in 19 additional subjects nerve potentials were recorded either from the high popliteal region or from the upper thigh.

Centripetal nerve volleys were frequently recorded at a stimulus intensity subthreshold for the reflex response both in normal subjects and spastic patients (Fig. 1). Nerve potentials were also recorded before the occurrence of the direct motor response in four of nine instances in which it had a lower 

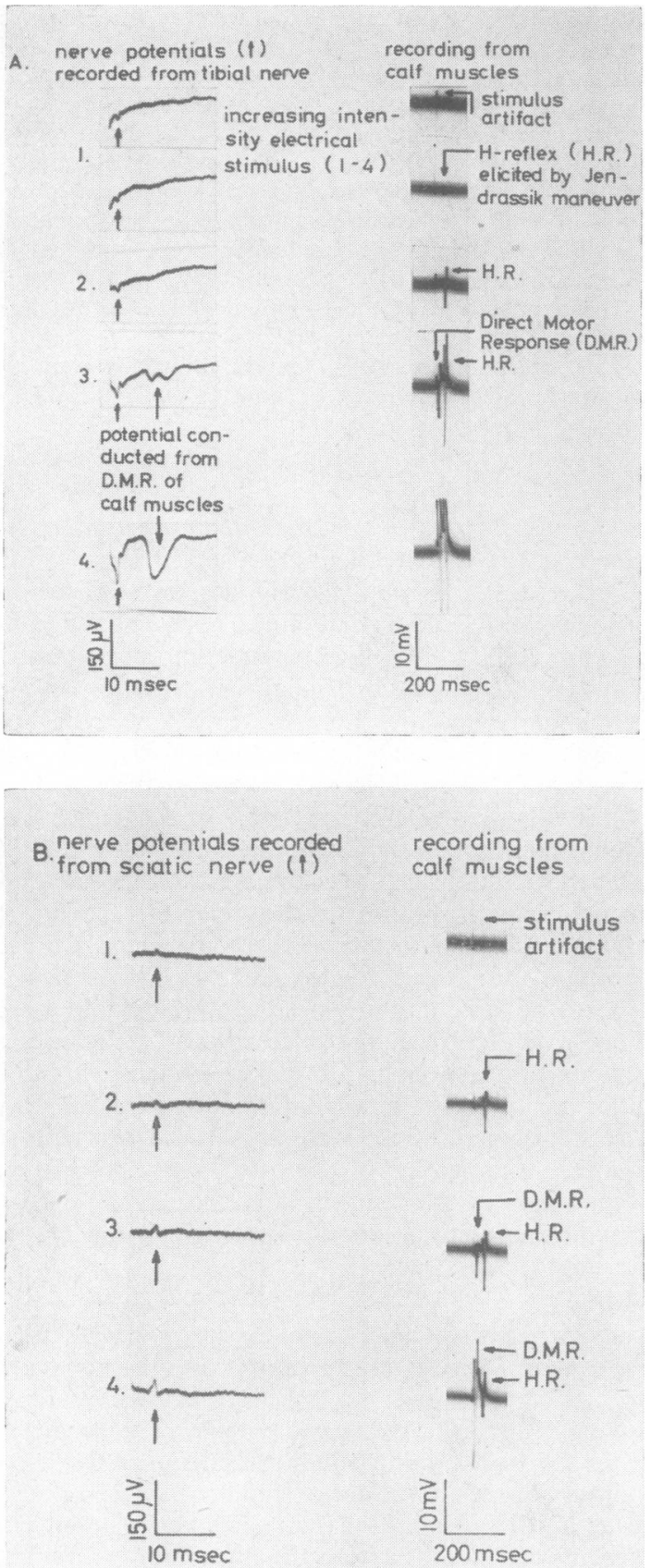

FIG. 1. Nerve potentials recorded in the high popliteal region (A) and in the upper thigh (B) on stimulation of the posterior tibial nerve in the popliteal fossa. The intensity of stimulation is progressively increased $(1 \rightarrow 4)$; and the response from the calf muscles is recorded simultaneously. threshold than the reflex. Nerve potentials were first $z$ recorded only with the appearance of the direct motor response in five instances. In one subject a nerve potential was recorded before the appearance of a low threshold reflex response at the popliteal site, whereas a nerve potential appeared only at higher stimulus intensity, with the direct motor $\stackrel{?}{?}$ response, at the sciatic site. The most likely explanation was that the sciatic recording electrodes were not 2 in proximity to the nerve, although changing the $\vec{F}$ electrode position did not alter the result.

A nerve potential subthreshold for the direct? motor response was found in four of seven subjects on stimulation of the musculocutaneous nerve and $\frac{\Phi}{\square}$ recording from the upper trunk of the brachial $\stackrel{\mathbb{\Omega}}{\complement}$ plexus (Fig. 2). In the biceps brachii muscle an $\%$ $\mathrm{H}$-reflex is an unusual occurrence.

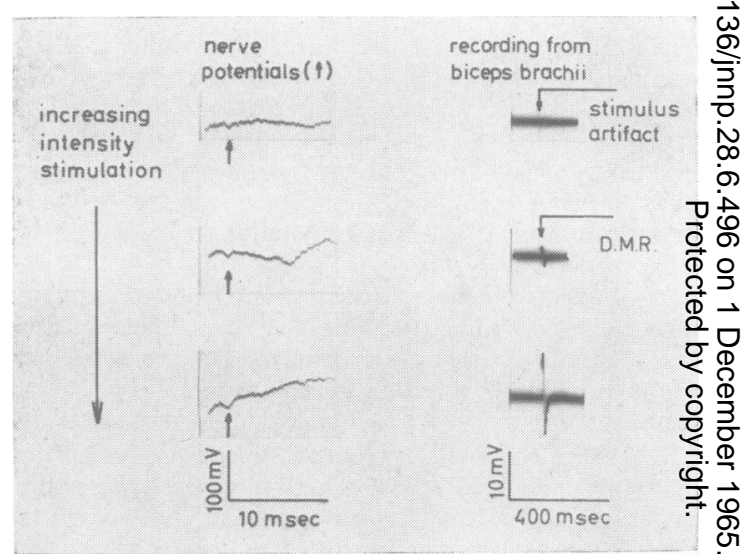

FIG. 2. Nerve potentials recorded from the upper trunk of the brachial plexus at Erb's point on stimulation of the musculocutaneous nerve in the upper arm. The direct motor response from the biceps brachii is recorded simultaneously.

The potentials recorded at or below threshold for $\overrightarrow{\overrightarrow{0}}$ the reflex and below threshold for the motor fibres are sensory in origin and include at least some proprioceptive afferents from the calf muscles. Complicating features were avoided such as stimulation of the peroneal nerve because it sometimes? produced nerve potentials at the recording sites. $\frac{5}{3}$ Stimulation of the posterior tibial nerve at the ankle was found to produce a nerve potential only at an $ᄋ$ intensity near maximal for the motor response in the plantar foot muscles. However, simultaneous 욱 recording from the plantar foot muscles showed no $\rightarrow$ activity during subthreshold stimulation at the popliteal region, indicating that the nerve potentials $N$ were unlikely to be related to excitation of the plantar ${ }^{\circ}$ nerves. Paraesthesiae extending into the foot some- : 
times occurred at or below threshold for the H-reflex, but this was not a regular feature and could occasionally be avoided by slight change in position of the stimulating electrodes. This indicates that at times fibres subserving sensibility may contribute to the nerve potentials. Stimulation of areas of the skin adjacent to the effective stimulating point with the same stimulus intensity resulted in no nerve potential. It will be shown that the conduction velocity of the nerve volley subthreshold for the motor fibres calculated between two widely separated recording sites was equivalent to that of the largest afferent proprioceptive fibres. Finally when the afferent volley reached a critical magnitude an H-reflex could be evoked by the Jendrassik manoeuvre, demonstrating that the lowest threshold proprioceptive afferents were contributing to the volley.

With increasing stimulus intensity the H-reflex first increased in size, and then decreased in size with the development of direct motor response. The nerve potential simultaneously recorded showed a progressive increase in amplitude (Fig. 1). It is likely that the increase in size of the nerve potential with increasing stimulus intensity is due to excitation of additional afferent fibres and to motor fibres antidromically stimulated, innervating the calf muscles; however, at high stimulus intensity the fibres of the posterior tibial nerve supplying the foot muscles made a small contribution to the increase in size of the nerve potential. At high intensity stimulation additional nerve potentials of longer latency were sometimes recorded. These later nerve potentials bore no relationship to the reflex per se and were probably due to stimulation of slower conducting fibres by the higher stimulus intensity.

With increasing stimulus intensity the latency of the nerve potentials generally showed little or no change. However, in some cases in which the potential was small and the start indistinct there was an apparent decrease in the latency at high intensity stimulation due to the distinct appearance of a small initial phase. In these instances measurement to the peak of the potential showed no change with increasing intensity of stimulation.

CONDUCTION VELOCITY DETERMINED BY CHANGES IN LATENCY OF THE H-REFLEX AND DIRECT MOTOR RESPONSE STIMULATED AT PROXIMAL AND DISTAL PARTS OF THE LIMB The mean conduction velocity of the afferent limb of the H-reflex stimulated at points separated by 23 to $28.5 \mathrm{~cm}$. was $62.0 \mathrm{~m}$./sec. (range $45-75 \mathrm{~m} . / \mathrm{sec}$.; S.D. 10.6) in the 12 subjects tested. The mean motor conduction velocity calculated by changes of the direct motor response stimulated at the same sites was $52.0 \mathrm{~m}$./ $/ \mathrm{sec}$. (range

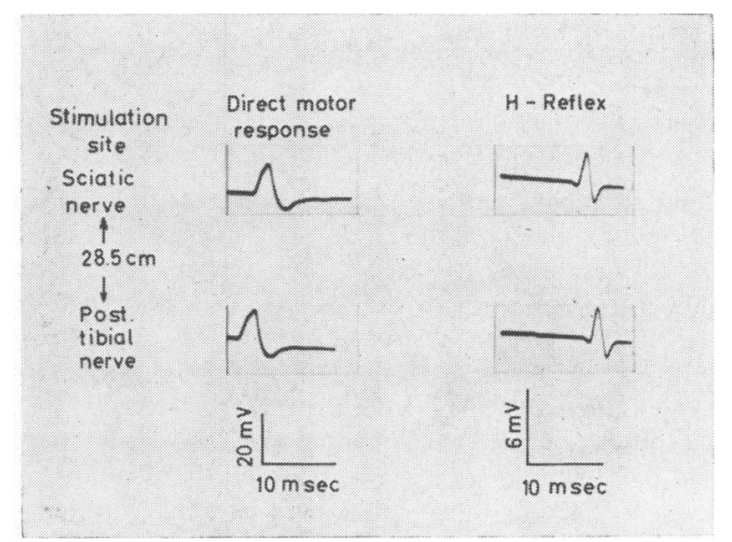

FIG. 3. Direct motor response and electrically evoked monosynaptic reflex (H-reflex) on stimulation in the popliteal fossa and in the upper thigh $28.5 \mathrm{~cm}$. between the points of stimulation.

40-69 m./sec.; S.D. $=9 \cdot 2$ ). In every subject the conduction velocity of the sensory limb of the reflex was greater than that of the largest motor fibres, with an average difference of $17 \%$ and a range of 10 to $22 \%$ (Fig. 3 ).

CONDUCTION VELOCITY DETERMINED BY NERVE POTENTIAL RECORDINGS The conduction velocity of the electrically evoked afferent volley subthreshold for the direct motor response, measured over a distance of 22 to $31 \mathrm{~cm}$., ranged from 62 to 80 $\mathrm{m}$./sec. in six subjects tested. In the determination the distance between the centre of the pair of bipolar electrodes in the popliteal and in the sciatic region was divided by the difference in latency to the start of the nerve action potential recorded at each site. In these six subjects and in an additional 15 subjects, the conduction velocity was also determined by the latency to the start of the potential recorded from the sciatic site and the distance between the stimulating cathode in the popliteal region to the middle of the bipolar sciatic recording electrodes. The mean conduction velocity was $66.7 \mathrm{~m}$./sec. (range 60 to $75 \mathrm{~m}$./sec., S.D. 6.2). In five instances a further determination was made by stimulating in the low popliteal and in the low sciatic regions, from 10 to $16 \mathrm{~cm}$. apart, and recording nerve potentials of similar appearance but altered latency from the sciatic bipolar electrodes. The conduction velocity between these stimulating points was of the same order as that determined by the latency from the stimulating cathode to the sciatic recording electrodes. The conduction velocity determined in the same subject both by changes in latency of the $\mathrm{H}$ reflex and by recording of nerve potentials showed no 
systematic difference and varied by no more than $10 \%$ in any of the three subjects so tested.

\section{DISCUSSION}

A centripetal nerve potential was recorded at or below threshold for the H-reflex and below threshold for the largest motor fibres. This afferent volley was shown to be conducted at a velocity equivalent to that of the largest proprioceptive fibres, and direct evidence that spindle afferents make an important contribution to the volley was provided by the elicitation of a reflex through the Jendrassik manoeuvre, with the intensity of stimulation and sensory volley unchanged and previously subthreshold for a reflex. Nevertheless it is not possible to conclude that only the largest afferent fibres of lowest threshold (group Ia fibres) are stimulated. It is probable that some group Ib fibres are excited and perhaps even a small number of group II fibres may be stimulated (Lloyd, 1943a; Eccles, 1957). Further, some cutaneous nerves in man have been reported to contain large diameter afferent fibres (Sunderland, Lavarack, and Ray, 1949), and paraesthesiae were sometimes experienced at low intensity stimulation, so that on occasion the largest cutaneous afferents may make a contribution to the volley.

The recording of a summated centripetal nerve potential from the popliteal and sciatic sites at a stimulus intensity subthreshold for the reflex response does not gainsay the conclusion that the nerve volley was made up primarily of spindle afferents. Lloyd (1943a) has shown that a reflex volley first occurred only when the dorsal root volley reached approximately $10 \%$ of maximum. Magladery et al. (1951b), however, recording from the spinal roots in man using intrathecal needle electrodes, found no afferent volley until the centrifugal volley was well developed.

It is distinctly unusual to find a low threshold H-reflex on stimulation of mixed nerves to muscles other than the calf in normal adult subjects. Yet a centrifugal volley was recorded subthreshold for excitation of motor fibres from the upper trunk of the brachial plexus on stimulation of the musculocutaneous nerve in the upper arm. Centripetal potentials have sometimes been recorded below threshold for the motor fibres on stimulation of the median or ulnar nerve at the wrist (Dawson and Scott, 1949) and of the anterior tibial nerve at the ankle (Gilliatt et al., 1961). However, in spastic patients, with increased central excitability, low threshold H-reflexes are more frequently recorded in muscles other than the calf. Nevertheless even in spastic patients a centripetal volley was found below threshold for the reflex. The absence of a monosynaptic discharge until a centripetal volley is $\bar{z}$ developed must reflect the need for a considerable $\stackrel{\mathbb{D}}{\perp}$ degree of summation within the motor neurone pool before a reflex discharge occurs.

That the more widespread elicitation of monosynaptic reflexes in spastic patients is unlikely to be related to alterations in the conditions of peripheral stimulation has been otherwise demonstrated in experiments in the decerebrate and spinal cat $\overrightarrow{2}$ (Gassel, unpublished data). In these experiments decerebrate cats were used with the lower extermity $\stackrel{P}{\rightarrow}$ immobilized and with stimulating electrodes fixed $\bar{c}$ in place on the anterior tibial nerve and fixed $\frac{\partial}{5}$ recording electrodes in the anterior tibial muscle. $\frac{\bar{\sigma}}{\vec{\sigma}}$ A low threshold H-reflex did not occur. However, $\stackrel{\mathbb{Q}}{\Omega}$ after spinalization in the lower thoracic region a reflex below threshold for the direct motor response was found in two of three experiments.

This introduces the concept of the extent to which $\overrightarrow{\vec{\omega}}$ the threshold of the reflex or motor response de- $\stackrel{\omega}{\stackrel{\omega}{\omega}}$ termined on excitation of a mixed nerve can be $\overrightarrow{\vec{J}}$ related directly to the relative size of the afferent 0 or motor fibres.

It is unlikely that fibre size alone determines the threshold of response on stimulation of the posteriot $\frac{\text { to }}{0}$ tibial nerve, percutaneously or with needle electrodes Slight changes in the position of the stimulating electrodes could alter the spectrum of results from one with a direct motor response at lowest threshol ${ }^{\circ}$ to one with a reflex response at threshold. The tibiad nerve supplying the calf muscles is not homogeneow in constitution. In cats the nerve fibres to the soleurs. muscles are considerably smaller than those to the $\vec{\theta}$ gastrocnemius '(Eccles and Sherrington, 1930), of and have a lower conduction velocity (Kuffler, Hunt, and Quilliam, 1951; Eccles, Eccles, and Lundberg, 1958); and the largest afferent fibres to the gastrocnemius are considerably larger than those to the tibialis posterior (Lloyd and Chang, 1948). Further, there is a degree of maintenance of fibre distribution for some distance proximal to the entry of a nerve branch (Sunderland and Bedbrook, 1949). Thus the position of the stimulating electrodes to the nerve fibres is an important factor. Nevertheless the usual findings of a lower threshold for the reflex response and a low threshold centripetal nerve volley on stimulation of the posterior tibial nerve, supported by the evidence of a higher conduction velocity of the afferent limb of the reflex than that of the largest motor fibres, leads to the conclusion that the largest afferent fibres are considerably larger than the motor fibres.

The occurrence of a direct motor response at lower threshold than the reflex response on stimulation of any peripheral nerve should not be interpreted to 0 indicate that the motor fibres are larger than the 
sensory. The factor of central excitability of the two-neurone arc is of critical importance.

The conduction velocity of the largest nerve fibres in the cat is approximately $120 \mathrm{~m}$. $/ \mathrm{sec}$. (Lloyd, 1943c; Hunt, 1954). Such high conduction velocities for the largest sensory or motor fibres have not been found in man by any of the various techniques employed for this determination. The conduction velocity in the largest motor fibres of the tibial nerve to the calf muscles averaged $52 \mathrm{~m}$./ $/ \mathrm{sec}$. in the present study, which is of the same order as that found in a previous study (Gassel and Trojaborg, 1964). The mean conduction velocity of the largest afferent proprioceptive fibres in the same stretch of nerve was 62 to $66 \mathrm{~m}$./ $/ \mathrm{sec}$. Mean values of approximately 50 to $60 \mathrm{~m}$./sec. have been reported in various studies of the conduction velocity of sensory and motor nerves in the upper extremity of man (Eichler, 1938; Dawson, 1956; Henricksen, 1958; Thomas, Sears, and Gilliatt, 1959; Mayer, 1963). The conduction velocity is related to the size of the nerve fibre and can be calculated by multiplying the external diameter in microns by a factor of 6 (Hursh, 1939). It is unlikely that any considerable difference in the spectrum of fibre diameters exists among the higher mammals, and the discrepancies in conduction velocity in cat and man are as yet unexplained.

It has been found that the afferent and efferent limb of the two-neurone arc in the nerve to the gastrocnemius in the cat has approximately the same conduction velocity (Lloyd, 1943b; Ekholm and Skoglund, 1964). In humans the H-reflex has been evoked at proximal and distal points in the popliteal region, approximately $10 \mathrm{~cm}$. apart, in determining the conduction velocity of the afferent limb of the two-neurone arc to the calf muscles. Magladery and McDougal (1950) found that the afferent conduction velocity over this rather restricted stretch of nerve was about $60 \mathrm{~m}$. $/ \mathrm{sec}$. and $33 \%$ greater than that of the motor fibres tested by supramaximal stimulation at the same popliteal sites. Mayer (1963) found the mean conduction velocity of the H-reflex was 61 to $71 \mathrm{~m}$./sec., over the short length of nerve in the popliteal region; and this was classified as a motor conduction velocity. He concluded that this velocity was significantly greater than that of the sensory nerve volley determined by maximal stimulation of the posterior tibial nerve at the ankle and recording from the tibial nerve at the knee. Magladery et al. (1951a) later reported that the conduction velocity of the afferent limb of the H-reflex was $10 \%$ greater than that of the motor limb on stimulation of the posterior tibial nerve in the popliteal fossa and recording the nerve potentials from the spinal roots using intrathecal electrodes. Liberson (1962) and Angel and Alston (1964) determined the 'afferent' conduction velocity of the late wave which follows the direct motor response in the plantar foot muscles on stimulation of the posterior tibial nerve at the ankle and knee. Angel and Alston found that the average conduction velocity of the 'spindle afferents' so determined was $43 \mathrm{~m}$./ $/ \mathrm{sec}$., the same value as that of the motor fibres. However, these late waves do not necessarily represent the monosynaptic reflex, and correspond more closely to the 'F-wave' which Magladery and McDougal (1950) concluded was polysynaptic in origin; although it has otherwise been found that the response is complicated by the recurrent discharge of motor neurones (Gassel, Marchiafava, and Pompeiano, 1965; Wiesendanger and Gassel, 1965). In calculating the conduction velocity of the ' $F$ waves' complexes evoked at proximal and distal sites must be of the same appearance and amplitude because of frequent variations in latency of complexes of different appearance (Gassel et al., 1965). There seems to be no reason a priori to conclude that the conduction velocity of the nerve fibres to the plantar foot muscles must perforce correspond to that to the calf muscles. The conduction velocity in the motor nerve fibres to these distal muscles is lower than that to the calf muscles (Gassel and Trojaborg, 1964). Further, the largest afferent nerve fibres to flexor muscles are smaller than those to extensor muscles (Lloyd and Chang, 1948; Rexed and Therman, 1948), although it should be noted that some of the plantar foot muscles act as physiological extensors. In the present study the conduction velocity of the afferent limb of the Hreflex determined over the long stretch of nerve extending from the low popliteal region to the upper thigh was, in each subject, greater than that of the motor fibres, calculated over the same stretch of nerve. The conduction velocity determined by changes in latency of the sensory nerve potential was of similar order to that calculated from changes in latency of the H-reflex.

\section{SUMMARY AND CONCLUSIONS}

A study was made of the relationship between nerve volleys and the threshold for the development of the monosynaptic reflex to the calf muscles in man. Nerve potentials were recorded with needle electrodes from the tibial nerve in the high popliteal region and from the sciatic nerve in the upper thigh, on stimulation of the posterior tibial nerve. It was found that slight changes in position of the stimulating electrodes altered the relative thresholds for the monosynaptic reflex and the direct motor response. A centripetal nerve volley was recorded frequently 
below threshold for either the monosynaptic reflex or the direct motor response. The proprioceptive fibres engaged in the two-neurone reflex were shown to make an important contribution to this nerve volley. A centripetal volley subthreshold for the reflex also occurred in spastic subjects.

Nerve potentials at a stimulus intensity subthreshold for the direct motor response were recorded from the upper trunk of the brachial plexus on stimulation of the musculocutaneous nerve. In the biceps brachii an H-reflex is an unusual occurrence.

These findings were interpreted as indicating that a considerable degree of spatial summation is necessary in the motor neurones before a reflex response develops. The implications of the results were considered for the evaluation of the relative sizes of sensory and motor fibres by the threshold of response on stimulation of a peripheral nerve.

The conduction velocity of the afferent limb of the monosynaptic reflex to the calf muscles, over the long stretch of nerve from the popliteal region to the upper thigh, was determined indirectly, by changes in latency of the monosynaptic reflex elicited at proximal and distal sites, and directly, from recordings of the afferent nerve volleys. The mean conduction velocity of the afferent limb was approximately $63 \mathrm{~m} . / \mathrm{sec}$., and $10 \%$ to $23 \%$ greater than that of the largest motor fibres, determined by changes in latency of the direct motor response evoked at the same sites.

\section{REFERENCES}

Angel, R. W., and Alston, W. (1964). Spindle afferent conduction velocity. Neurology (Minneap.), 14, 647-676.

Bolzani, L., and Sala, E. (1958). Sul comportamento dei riflessi $H$ ed F a diverse frequenze di stimolazione. Schweiz. Arch. Neurol. Psychiat., 82, 189-195.

Dawson, G. D. (1956). The relative excitability and conduction velocity of sensory and motor nerve fibres in man. J. Physiol. (Lond.), 131, 436-451.

- and Scott, J. W. (1949). The recording of nerve action potentials through skin in man. J. Neurol. Neurosurg. Psychiat., 12, 259-267.

Eccles, J. C. (1957). The Physiology of Nerve Cells. Johns Hopkins Press, Baltimore.

-, Eccles, R. M., and Lundberg, A. (1958). The action potentials of the alpha motoneurones supplying fast and slow muscles. J. Physiol. (Lond.), 142, 275-291.

- and Sherrington, C. S. (1930). Numbers and contraction values of individual motor-units examined in some muscles of the limb. Proc. roy. Soc. B, 106, 326-357.

Eichler, W. (1938). Uber die Ableitung der Aktionspotentiale vom menschlichen Nerven in situ. $Z$. Biol., 98, 182-214.

Ekholm, J., and Skoglund, S. (1964). Possible factors influencing the demonstration of post-tetanic potentiation of the H-reflex as studied in the cat. Exp. Neurol., 9, 183-197.

Gassel, M. M. (1964). A test of nerve conduction to muscles of the shoulder girdle as an aid in the diagnosis of proximal neurogenic and muscular disease. J. Neurol. Neurosurg. Psychiat. 27, 200-205.
Gassel, M. M., Marchiafava, P. L., and Pompeiano, O. (1965). Modulation of the recurrent discharge of alpha motoneurones in decerebrate and spinal cats. Arch. ital. Biol., 103, 1-24.

- , and Trojaborg, W (1964). Clinical and electrophysiologicalo study of the pattern of conduction times in the distribution? of the sciatic nerve. J. Neurol. Neurosurg. Psychiat., 27, 351357.

Gilliatt, R. W. Goodman, H. V., and Willison, R. G. (1961). The T recording of lateral popliteal nerve action potentials in man Ibid., 24, 305-318.

Henricksen, J. D. (1958). Conduction velocity of motor nerves in normal subjects and patients with neuromuscular disease. M.S. Thesis, University of Minnesota.

Hunt, C. C. (1954). Relation of function to diameter in afferent fibersof muscle nerves. J. gen. Physiol., 38, 117-131.

Hursh, J. B. (1939). Conduction velocity and diameter of nerve fibers. Amer. J. Physiol., 127, 131-139.

Kuffler, S. W., Hunt, C. C., and Quilliam, J. P. (1951). Function of medullated small-nerve fibers in mammalian ventral roots: efferent muscle spindle innervation. J. Neurophysiol., 14, 29-54. $\frac{\text { S }}{\partial}$

Liberson, W. T. (1962). Monosynaptic reflexes and their clinical sig- $\mathbb{Q}$ nificance. In Progress in Electromyography, edited by P. Pinelli, Electroenceph. clin. Neurophysiol., suppl. 22, pp. ڤ్ 79-89.

Lloyd, D. P. C. (1943a). Reflex action in relation to pattern and peri- $\overrightarrow{0}$ pheral source of afferent stimulation. J. Neurophysiol., 6, 111-

- $\quad 119$. . hind limb reflexes in cat. Ibid., 6, 293-315.

- (1943c). Conduction and synaptic transmission of the reflex response to stretch in spinal cats. Ibid., 6, 317-326.

—, and Chang, H. T. (1948). Afferent fibres in muscle nerves. Ibid., 11, 199-207.

Magladery, J. W., and McDougal, D. B. Jr. (1950). Electrophysiolcgical studies of nerve and reflex activity in normal man I. Identification of certain reflexes in the electromyogram and the conduction velocity of peripheral nerve fibres. Bull. Joknss Hopk. Hosp., 86, 265-290.

, Porter, W. E. Park, A. M., and Teasdall, R. D. (1951a). Elect原physiological studies of nerve and reflex activity in nornal man. IV. The two-neurone reflex and identification of certain action potentials from spinal roots and cord. Ibid., 88, 499-5T. Teasdall, R. D., Park, A. M., and Porter, W. E. (1951Q. Electrophysiological studies of nerve and reflex activity on normal man. V. Excitation and inhibition of two-neurofe $\mathbb{D}$ reflexes by afferent impulses in the same nerve trunk. Ibid., 520-537.

Mayer, R. F. (1963). Nerve conduction studies in man. Neurology (Minneap.), 13, 1021-1030.

Pinelli, P. and Valle, M. (1960). Studio fisiopatologico dei rifless muscolari nelle parese spastiche, Arch. Sci. med., 110, 77-202.

Rexed, B., and Therman, P. O. (1948). Calibre spectra of motor and sensory nerve fibres to flexor and extensor muscles. J. Neurophysiol., 11, 133-139.

Sunderland, S., and Bedbrook, G. M. (1949). The cross-sectional area of peripheral nerve trunks occupied by the fibres representing $\mathbb{Q}$ individual muscular and cutaneous branches. Brain, 72, 0 613-624.

- Lavarack, J. O., and Ray, L. J. (1949). The caliber of nerve $\overline{\mathrm{O}}$ fibers in human cutaneous nerves. J. comp. Neurol., 91, 87-101.

Teasdall, R. D., Park, A. M., Languth, H. W., and Magladery, $\vec{P}$ J. W. (1952). Electrophysiological studies of reflex activity in patients with lesions of the nervous system. II. Disclosure of normally suppressed monosynaptic discharge of spinal motoneurones by lesions of lower brain-stem and spinal cord. Bull. Johns Hopk. Hosp., 91, 245-256.

Thomas, P. K., Sears, T. A., and Gilliatt, R. W. (1959). The range of 0 conduction velocity in normal motor nerve fibres to the small 3 muscles of the hand and foot. J. Neurol. Neurosurg. Psychiat., 22, $175-181$.

Wiesendanger, M., and Gassel, M. M. (1965). Recurrent and reflex discharges in plantar muscles of the cat. Acta physiol. scand., in the press. 